\title{
Synthetic MRI for Clinical Neuroimaging: Results of the Magnetic Resonance Image Compilation (MAGiC) Prospective, Multicenter, Multireader Trial
}

(D)L.N. Tanenbaum, (D)A.J. Tsiouris, (D)A.N. Johnson, (D)T.P. Naidich, (D) M.C. DeLano, (DE.R. Melhem, (DP. Quarterman, (D) S.X. Parameswaran, (D)A. Shankaranarayanan, (DM. Goyen, and (D)A.S. Field

\begin{abstract}
BACKGROUND AND PURPOSE: Synthetic MR imaging enables reconstruction of various image contrasts from 1 scan, reducing scan times and potentially providing novel information. This study is the first large, prospective comparison of synthetic-versus-conventional MR imaging for routine neuroimaging.
\end{abstract}

MATERIALS AND METHODS: A prospective multireader, multicase noninferiority trial of 1526 images read by 7 blinded neuroradiologists was performed with prospectively acquired synthetic and conventional brain MR imaging case-control pairs from 109 subjects (mean, $53.0 \pm 18.5$ years of age; range, 19-89 years of age) with neuroimaging indications. Each case included conventional T1- and T2-weighted, T1 and T2 FLAIR, and STIR and/or proton density and synthetic reconstructions from multiple-dynamic multiple-echo imaging. Images were randomized and independently assessed for diagnostic quality, morphologic legibility, radiologic findings indicative of diagnosis, and artifacts.

RESULTS: Clinical MR imaging studies revealed 46 healthy and 63 pathologic cases. Overall diagnostic quality of synthetic MR images was noninferior to conventional imaging on a 5-level Likert scale $(P<.001$; mean synthetic-conventional, $-0.335 \pm 0.352 ; \Delta=0.5$; lower limit of the $95 \% \mathrm{Cl},-0.402$ ). Legibility of synthetic and conventional morphology agreed in $>95 \%$, except in the posterior limb of the internal capsule for T1, T1 FLAIR, and proton-density views (all, $>80 \%$ ). Synthetic T2 FLAIR had more pronounced artifacts, including $+24.1 \%$ of cases with flow artifacts and $+17.6 \%$ cases with white noise artifacts.

CONCLUSIONS: Overall synthetic MR imaging quality was similar to that of conventional proton-density, STIR, and T1- and T2-weighted contrast views across neurologic conditions. While artifacts were more common in synthetic T2 FLAIR, these were readily recognizable and did not mimic pathology but could necessitate additional conventional T2 FLAIR to confirm the diagnosis.

ABBREVIATIONS: $\kappa=$ Kappa statistic; MAGiC = MAGnetic resonance image Compilation; $M D M E=$ multiple-dynamic multiple-echo; $P D=$ proton density

$\mathbf{S}$ ynthetic MR imaging uses quantitative probing of multiple physical properties to reconstruct multiple contrasts from 1 scan. Parameters like TR, TE, and TI can be modified with mathematic inferences rather than being predetermined. ${ }^{1-3}$ The speed of diagnostic brain studies can thus be reduced to only about 5

Received December 2, 2016; accepted after revision February 2, 2017.

From Lenox Hill Radiology (L.N.T.), RadNet Inc, New York, New York; Department of Radiology (A.J.T.), Weill Cornell Medical Center, New York, New York; Department of Technical Communication (A.N.J.), Science and Healthcare, Texas Tech University, Lubbock, Texas; Technology and Medical Innovation Organization (A.N.J., S.X.P.), Healthcare Imaging-MRI (P.Q.), Healthcare Imaging-MRI Neuro Applications (A.S.), and Medical Affairs (M.G.), GE Healthcare, Milwaukee, Wisconsin; Department of Neuroradiology (T.P.N.), The Mount Sinai Hospital, New York, New York; Division of Radiology and Biomedical Imaging (M.C.D.), Michigan State University, Advanced Radiology Services, PC, and Spectrum Health, Grand Rapids, Michigan; Department of Diagnostic Radiology and Nuclear Medicine (E.R.M.), University of Maryland School of Medicine, Baltimore, Maryland; and Department of Radiology (A.S.F.), University of Wisconsin School of Medicine and Public Health, Madison, Wisconsin.

This work was supported by GE Healthcare to participating institutions. minutes with synthetic MR imaging. ${ }^{4}$ This advancement may help improve throughput and reduce rescanning, while also providing quantitative information of research interest. ${ }^{4-6}$

Clinical studies of synthetic MR imaging are highly heterogeneous in that they examine a variety of conditions with widely varying scan parameters, with a paucity of large, randomized trials to inform clinical usage. ${ }^{3,6}$ Blystad et $\mathrm{al}^{5}$ (2012) reported that synthetic images had diagnostic utility similar to that of conventional imaging series, though with some quality issues like granulation and contrast particularly apparent in FLAIR views. Other studies reported good quality and contrast

Please address correspondence to Lawrence Tanenbaum, MD, 400 E 66th St, NY, NY 10065; e-mail: Nuromri@radnet.com; @nuromri

- Indicates open access to non-subscribers at www.ajnr.org

$\equiv$ Indicates article with supplemental on-line tables.

Indicates article with supplemental on-line photos.

http://dx.doi.org/10.3174/ajnr.A5227

AJNR Am J Neuroradiol 38:1103-10 Jun 2017 www.ajnr.org 
for synthetic images among certain indications, such as multiple sclerosis, brain metastasis, ${ }^{6,7}$ and myelination patterns. ${ }^{8}$ Because image quality endpoints are reliant on reader judgment (reported to have up to $41 \%$ variability and only fair-tomoderate interrater agreement ${ }^{9,10}$ ) and scanning conditions, drawing clinically relevant inferences from diverse small trials is challenging. Furthermore, the broad diversity of both healthy and pathologic morphologic variants encountered in routine neuroimaging necessitates more robust clinical studies of synthetic MR imaging for clinical neuroimaging.

This study was designed to compare the overall image quality of synthetic MR imaging with conventional MR imaging in a general neuroimaging population. Secondary aims included legibility of anatomic and morphologic features, artifact prevalence, and diagnostic performance across a range of cases helpful in informing clinical usage and adoption of synthetic MR imaging.

\section{MATERIALS AND METHODS}

\section{Participants and Clinical Assessments}

Subjects $(n=117)$ were enrolled prospectively into a multireader multicenter case-control study across 6 hospitals from November 2015 to January 2016 (ClinicalTrials.gov Identifier NCT02596854). Of these, all complete cases ( $n=109 ; 45$ men, 64 women; mean, $53.0 \pm 18.5$ years of age; range, $19-89$ years) with synthetic and conventional (control) acquisitions were read. Subjects were 18 years of age or older with clinical indications for neuroimaging and without contraindications to MR imaging or previously diagnosed congenital conditions or extensive trauma prohibiting scanning. Governing ethics committees at each site approved this study, and subjects provided written informed consent.

\section{Image Acquisition}

Images were prospectively acquired by using a fixed set of scanning parameters closely approximating current standard of care brain MR imaging (as detailed for $1.5 \mathrm{~T}$ and 3T scanners in Online Table 1). First, conventional images were acquired by using conventional 2D axial plane T1- and T2-weighted, T1 and T2 fluid-attenuated inversion recovery, short tau inversion recovery, and proton density (PD) sequences. Then, a multiple-dynamic multiple-echo (MDME) sequence was performed for synthetic reconstruction, for a complete conventional and synthetic casecontrol series. MDME uses a repeat version of the same gradientreversal process used to create a single gradient-echo to produce additional gradient-echoes after a single radiofrequency pulse. This is known as multiple (or dual) echo gradient-echo, which is possible when complete loss of the transverse magnetization by $\mathrm{T}^{*}$ relaxation has not yet occurred. Because MDME is a quantitative sequence, it enables absolute quantification of tissue physical properties, like longitudinal $R_{1}$ relaxation rate, transverse $R_{2}$ relaxation rate, and $\mathrm{PD}$ independent of the scanner settings. MDME parameters acquired in 1 scan are used in synthetic imaging to calculate pixel intensity, producing an appearance similar to that of conventional MR images with modifiable TE, TR, and TI. ${ }^{5,11}$ Thus, synthetic (based on MDME) and conventional T1, T2, T1 FLAIR, T2 FLAIR, PD, and STIR contrast views were col- lected. MDME data were reconstructed outside the clinical care environment by using MAGnetic resonance image Compilation (MAGiC) software on a 64-bit Advantage Workstation (GE Healthcare, Milwaukee, Wisconsin). No errors were logged during processing, and the average processing time was approximately 2 minutes per case. Scan duration, subject disposition, and imaging results were recorded for each case.

The site-determined diagnosis was recorded on the basis of the results of MR imaging studies and work-up performed according to the standard of care by clinical neuroradiologists. The sites reported the reference (site-determined diagnosis) by using the same scale as the study readers, which reports normal or $\geq 1$ pathologic subtype adapted from Osborn et al $(2010)^{12}$ : 1) traumatic, complex, indeterminate, or other condition or injury; 2) congenital malformation; 3) ischemic or hemorrhagic stroke; subarachnoid hemorrhage/aneurysm; 4) vascular malformation; 5) neoplasm/primary neoplastic cysts; 6) infectious/demyelinating disease; or 7) metabolic/degenerative disorders.

\section{Radiologic Assessments}

Synthetic and conventional images sets were randomized and assessed by 7 blinded independent neuroradiologists $(>10$ years' experience) on standard imaging workstations. Casecontrol pairs from the same subject were separated and read across 2 sessions, separated by a 4 -week memory-washout period. Each read included either all synthetic or all conventional contrast views from a case. Overall diagnostic image quality was rated (considering all available contrast views) on a 5-point Likert-type scale: 5 = excellent (acceptable for diagnostic use), $4=$ good (acceptable for diagnostic use), $3=$ acceptable (acceptable for diagnostic use but with minor issues), 2 = poor (not acceptable for diagnostic use), or $1=$ unacceptable (not acceptable for diagnostic use). Ratings of $\geq 3$ were considered acceptable overall. For image sets rated as unacceptable ( 1 or 2 ), the rationale was recorded as "open text." Readers also recorded radiologic findings indicative of a diagnosis with corresponding Osborn classifications.

For each contrast view, readers rated the legibility (or visibility of margins and structures associated with key anatomic/morphologic features) of anatomies defined a priori. Legibility ratings supplemented overall image-quality data, which consider all regions of the brain, as a means of providing specific information about anatomic regions in brain imaging. Each anatomy was rated on a binary scale (legible/illegible), including the following: central sulcus, head of the caudate nucleus, posterior limb of the internal capsule, cerebral peduncle, middle cerebellar peduncle, and cervicomedullary junction. Readers recorded whether any of the following artifacts were present: ${ }^{13}$ low signal-to-noise, motion and section issues, infolding or wrap-around, white pixel or spike noise, phase encoding, flow, contrast-to-noise, low image resolution, or blurring. Readers could provide free text comments on any other observations.

\section{Statistical Analysis}

Statistical analysis was performed in SAS 9.2 (SAS Institute, Cary, North Carolina), and sample size was calculated in PASS12 
Diagnostic image-quality ratings by static field strength of scanner and overall ${ }^{\mathrm{a}}$

\begin{tabular}{|c|c|c|c|c|c|c|}
\hline \multirow[b]{2}{*}{ Diagnostic Quality ${ }^{\mathrm{b}}$} & \multicolumn{2}{|c|}{ 1.5T Scanner } & \multicolumn{2}{|c|}{ 3T Scanner } & \multicolumn{2}{|c|}{ Overall $(1.5 T+3 T)$} \\
\hline & Syn $(N=392)$ & Con $(N=392)$ & Syn $(N=371)$ & Con $(N=371)$ & Syn $(N=763)$ & Con $(N=763)$ \\
\hline Acceptable for diagnostic use $(3,4,5)$ & $380(97 \%)$ & $387(99 \%)$ & $354(95 \%)$ & $358(96 \%)$ & $734(96 \%)$ & 745 (98\%) \\
\hline Excellent (rated 5) & $64(16 \%)$ & $183(47 \%)$ & $37(10 \%)$ & $103(28 \%)$ & $101(13 \%)$ & $286(37 \%)$ \\
\hline Good (rated 4) & $266(68 \%)$ & $170(43 \%)$ & $230(62 \%)$ & $199(54 \%)$ & $496(65 \%)$ & $369(48 \%)$ \\
\hline Acceptable (rated 3) & $50(13 \%)$ & $34(9 \%)$ & $87(23 \%)$ & $56(15 \%)$ & $137(18 \%)$ & $90(12 \%)$ \\
\hline Unacceptable for diagnostic use $(1,2)$ & $12(3 \%)$ & $5(1 \%)$ & $17(5 \%)$ & $13(4 \%)$ & $29(4 \%)$ & $18(2 \%)$ \\
\hline Poor (rated 2) & $10(3 \%)$ & $5(1 \%)$ & $16(4 \%)$ & $12(3 \%)$ & $26(3 \%)$ & $17(2 \%)$ \\
\hline Unacceptable (rated 1) & $2(1 \%)$ & 0 & $1(0.3 \%)$ & $1(0.3 \%)$ & $3(0.4 \%)$ & $1(0.1 \%)$ \\
\hline
\end{tabular}

Note:-Syn indicates synthetic MR imaging; Con = conventional MR imaging (control).

${ }^{\text {a }}$ All data are shown as $n(n / \mathrm{N} \%)$, where $n$ is the count and $N$ is the total reads per category (defined in the upper row of this table).

${ }^{b}$ Five-point scale.

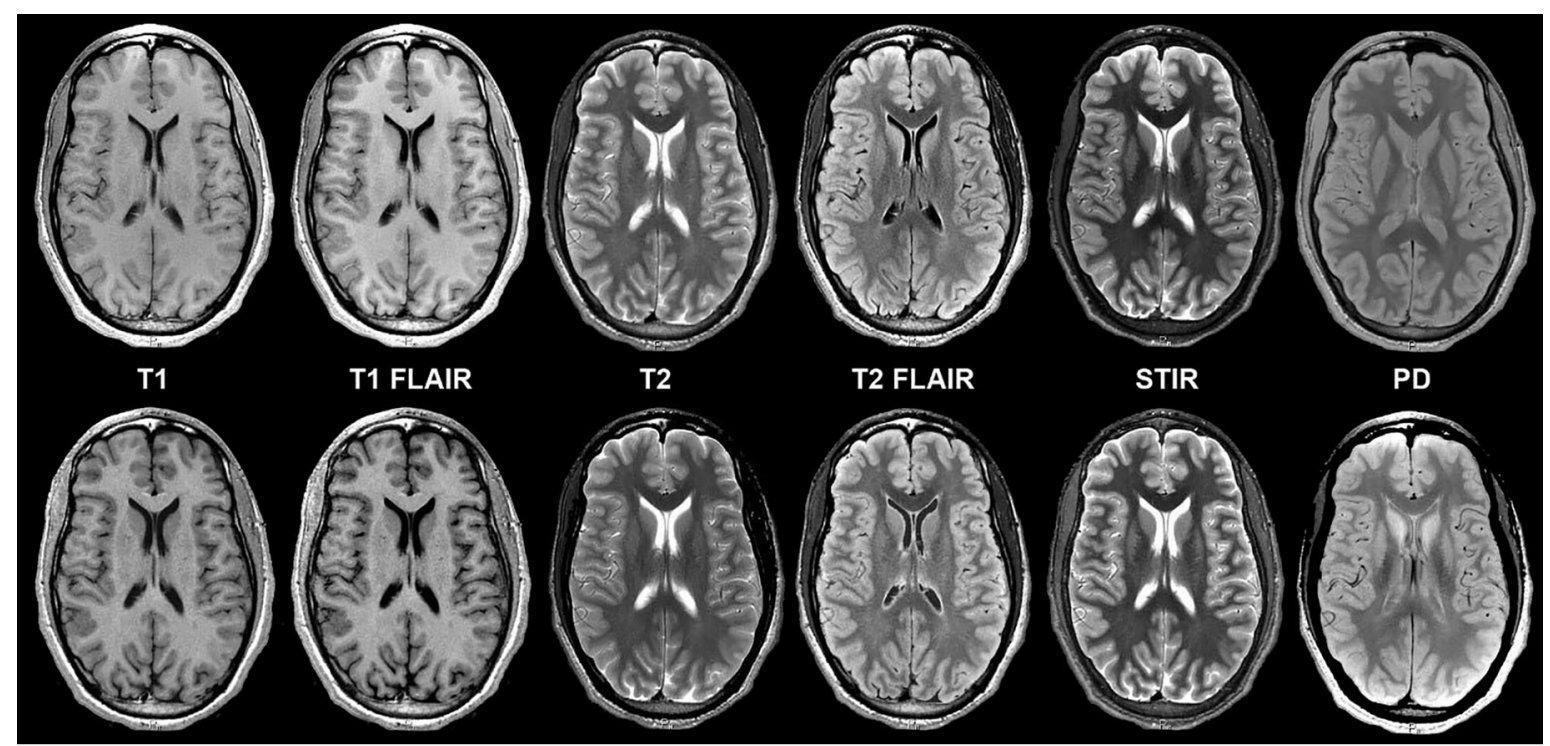

FIG 1. Axial synthetic and conventional 3T MR imaging of a normal brain. Conventional (upper row) and synthetic (lower row) image sets exhibit similar legibility and quality. Slight differences in contrast levels are apparent, which do not adversely impact the diagnostic utility of images, particularly between T1 FLAIR and T2 FLAIR views.

(NCSS Statistical Software, Kaysville, Utah). Per the prospective statistical plan to determine noninferiority, a Wilcoxon signed rank test was used to determine noninferiority of synthetic-toconventional MR imaging in terms of the overall diagnostic image quality score, by using a 1 -sided $\alpha=.025$ test with a noninferiority margin of $\Delta=.5$ with a 5 -level Likert scale. The primary hypothesis is 1-sided and can be stated as $H_{0}: S \leq-\Delta$ and $H_{\mathrm{A}}: S>$ $-\Delta$, where the $S$ is the median difference of overall diagnostic image quality across readers for synthetic-versus-conventional MR imaging, in which noninferiority is established by rejecting the null hypothesis. The margin $(\Delta)$ of .5 was determined statistically on the basis of the population and was confirmed by clinical estimates from prior research ${ }^{5}$ and institutional pilot data, in accordance with recommendations for determination of noninferiority margins described in the US Food and Drug Administration Guidance for Industry: Non-Inferiority Clinical Trials to Establish Effectiveness (2016) (https://www.fda.gov/ downloads/Drugs/GuidanceComplianceRegulatoryInformation/ Guidances/UCM202140.pdf) and trial designs for noninferiority testing in radiology reviewed by Ahn et al (2012). ${ }^{14}$ Descriptive statistics were used to summarize secondary endpoints of anatomic/ morphology legibility by anatomic region, artifact prevalence, and diagnostic performance (sensitivity/specificity) by the Osborn classi- fication. Interrater reliability between readers was assessed by kappa ( $\kappa)$ statistic.

\section{RESULTS}

\section{Overall Diagnostic Quality}

Each of 7 blinded neuroradiologists read all 109 clinically acquired case-control image sets (109 synthetic and 109 conventional) for a total of 1526 reads (763 synthetic and 763 conventional reads). Of these, 56/109 were acquired on $1.5 \mathrm{~T}$ static field strength scanners and 53/109 were acquired on 3T scanners. Because no significant differences for 5-level image quality scores (acceptable $=3,4$, or 5 versus unacceptable $=1$ or 2 ) were observed on the basis of scanner static field strength (1.5T or T) or acquisition site $(P>.05$ with a 2 -tailed $t$ test $)$, results were pooled for analysis. The duration of scanning was recorded, with a singleacquisition sequence for synthetic reconstruction requiring 5 minutes 36 seconds on $1.5 \mathrm{~T}$ scanners and 5 minutes 4 seconds on 3T scanners (On-line Table 1).

Considering all contrast views, 734 (96\%) synthetic cases and $745(98 \%)$ conventional cases were rated as acceptable ( $\geq 3$ on a 5 -point scale) (Table). Figure 1 shows comparable synthetic and conventional case-control images from a normal (no pathology present) brain by contrast view. Figures $2-5$ show case-control 


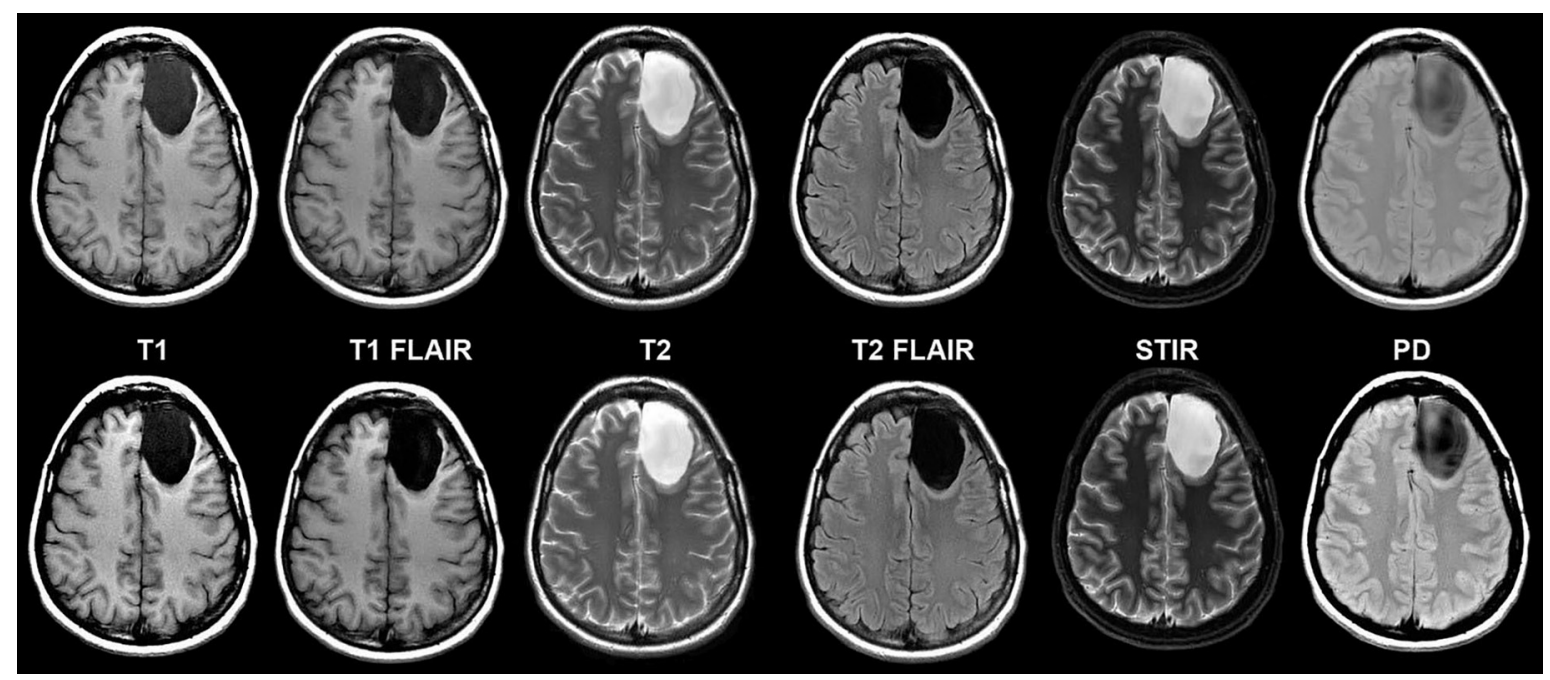

FIG 2. Left frontal lobe cystic tumor on axial synthetic and conventional 3T MR imaging in a 31-year-old woman. Conventional (upper row) and synthetic (lower row) image sets exhibit similar legibility and quality.

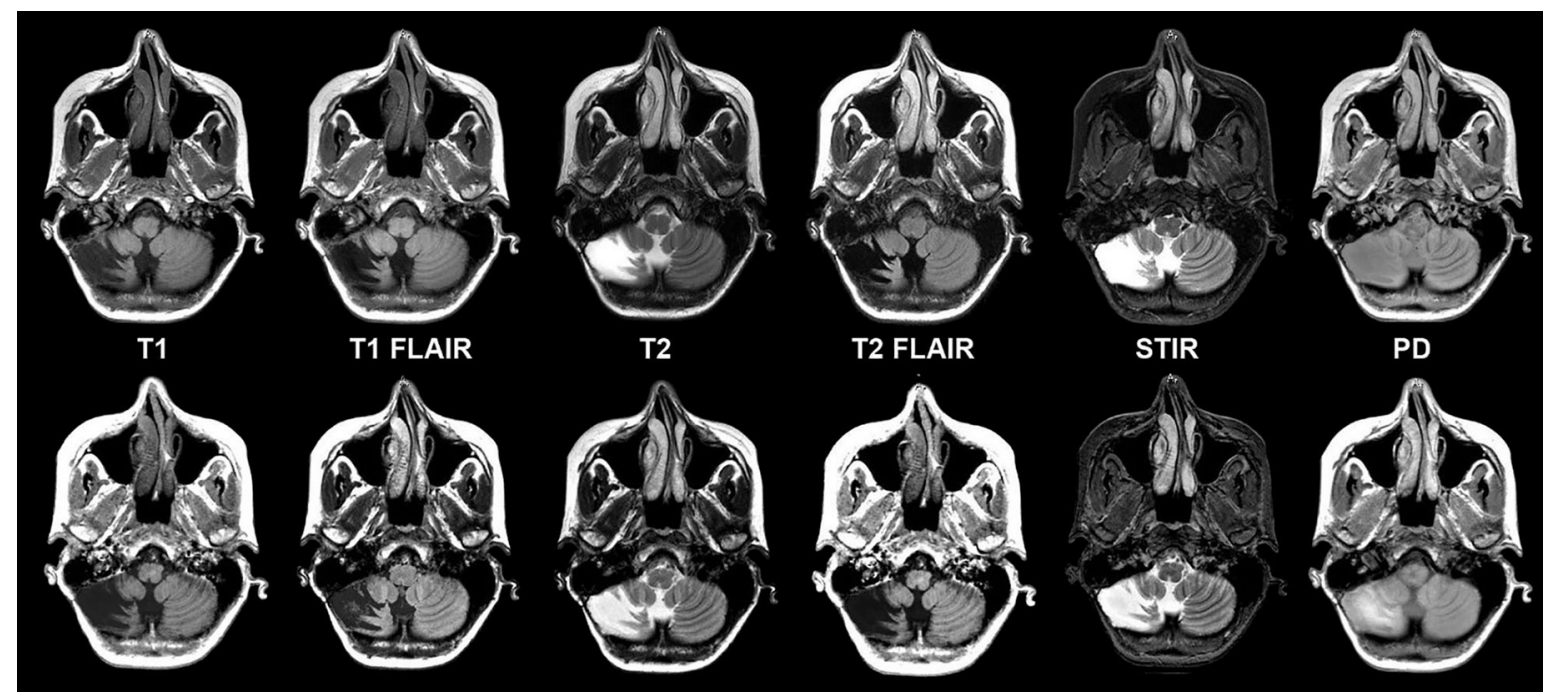

FIG 3. Chronic right cerebellar infarction in a 37-year-old woman on axial synthetic and conventional 3T MR imaging. Conventional (upper row) and synthetic (lower row) image sets exhibit similar legibility and quality.

images across a range of brain pathologies (continued in On-line Figs 1-3). Overall diagnostic image quality of synthetic images was statistically noninferior to conventional images, with a mean difference (synthetic-conventional) across readers of $-0.335 \pm$ 0.352 with a lower limit of the (1-sided) $95 \%$ CI of -0.402 (median, -0.428; minimum, -1.286 ; and maximum, $0.714 ; P<$ .001). Among synthetic images rated as poor or unacceptable (1 or 2 on a 5 -point scale), the most common quality issue was patient motion in synthetic image sets owing to generating from a single acquisition (where a single motion event propagates across all reconstructed contrast views).

\section{Legibility of Anatomic/Morphologic Features}

Anatomic/morphologic features were visualized and rated as legible in synthetic and conventional imaging for $\geq 98 \%$ of regions across contrast views, except in the cervicomedullary junction rated at $96 \%$ on both synthetic and conventional imaging (Online Table 2). For synthetic and conventional pairs from the same subject, readers agreed for $\geq 95 \%$ of anatomic/morphologic regions across contrast views, except in the posterior limb of the internal capsule for T1, T1 FLAIR, and PD views ( $>80 \%$ agreement). Notably, 6 of 7 readers had agreement of $99 \%-100 \%$ for T1 FLAIR, with 1 reader as an outlier at $89 \%$, possibly related to experience. Further study will be needed to investigate the influence of experience on reading synthetic images and possible training solutions.

\section{Artifacts Occurrence and Characterization}

Fewer artifacts (all characterizations) were identified in synthetic than in conventional imaging for T1-weighted (9.2\%), STIR (24.8\%), and PD (1.1\%) contrast views (On-line Table 3). Synthetic images had more artifacts overall on the T2-weighted (5.0\%), T1 FLAIR (17.9\%), and T2 FLAIR (49.3\%) contrast views (On-line Table 3). Phase-encoding artifacts were less frequent in synthetic STIR images (27.2\%) and synthetic T1 contrast views $(13.0 \%)$. Synthetic contrast views were more likely to contain 


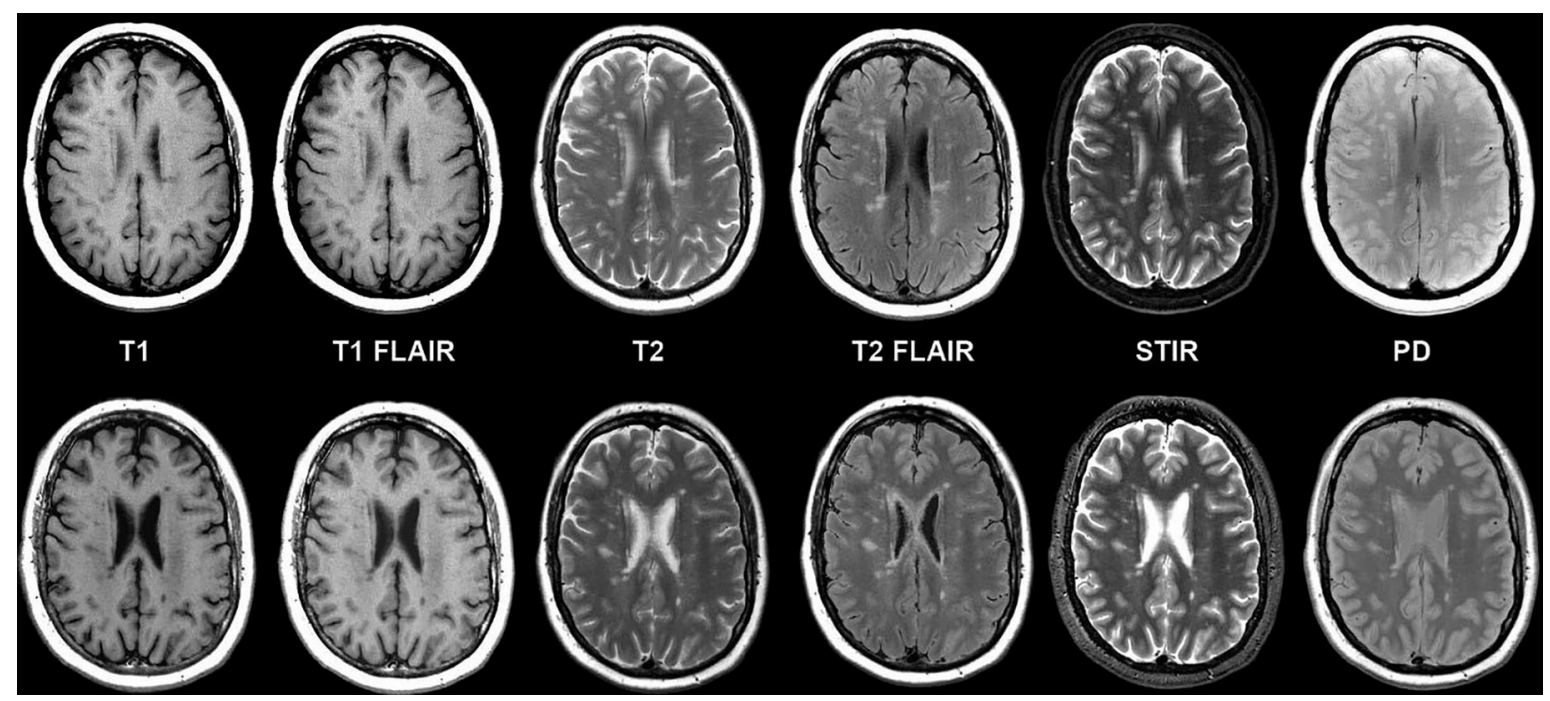

FIG 4. Multiple sclerosis in axial synthetic and conventional 3T MR imaging in a 58-year-old woman. Multifocal demyelinating lesions are apparent within the cerebral white matter; these lesions appear similar on conventional (upper row) and synthetic (lower row) image sets. A slight misregistration is apparent due to patient motion between the MDME scan (used for synthetic reconstruction, lower row) and the comparable conventional scan acquired in the study (upper row). While misregistration due to motion can pose challenges in conventional serial acquisitions due to partial section differences in images across contrast views, synthetic reconstruction inherently prevents misregistration across synthetic contrast views.

white pixels/spike noise artifacts across contrast views (except $\mathrm{PD})$, and flow artifacts were more common in synthetic views, most notably in the synthetic T1-weighted (13.0\%), T1 FLAIR (22.1\%), and T2 FLAIR (24.1\%) contrast views.

Readers identified relatively more artifacts among synthetic T2 FLAIR contrast views compared with other synthetic and conventional contrast views. Synthetic T2 FLAIR showed 24.1\% more flow artifacts, $17.6 \%$ more white noise artifacts, and 59.2\% more artifacts marked as "other" compared with conventional views. Examination of reader free text comments revealed that artifacts marked as "other" primarily described localized, granulated hyperintensities apparent in the margins only in synthetic T2 FLAIR contrast views (Fig 6). These artifacts were recognizable by a distinct pixelated appearance and a tendency to occur along tissueCSF boundaries only in T2 FLAIR views in otherwise unremarkable image sets. Readers reported that synthetic T2 FLAIR may have some diagnostic limitations in practice, which could necessitate a conventional T2 FLAIR scan. However, owing to the nature of synthetic imaging (which results in a full range of possible contrast views for cross-comparison), neuroradiologists were readily able to distinguish T2 FLAIR artifacts from pathology, without impacting diagnostic utility.

\section{Diagnostic Performance}

Overall interrater agreement ( $\kappa$ correlation coefficient) for pathology detection was 0.502 for synthetic images and slightly higher at 0.605 for conventional images. Across the 7 readers (1526 total reads, including 763 synthetic and 763 conventional pairs), overall sensitivity for correct identification of pathology ranged from 60.32 (95\% CI, 47.20-72.43) to 95.24 (95\% CI, 86.71-99.01) among readers for conventional versus 55.56 (95\% CI, 42.49-68.08) to 96.83 (95\% CI, 89.00-99.61) among readers for synthetic imaging, with the site-determined diagnosis as the criterion standard. On the basis of clinically confirmed diagnoses reported by the site (based on clinical MR imaging studies and, when necessary, additional follow-up or laboratory testing), the study included 46 healthy and 63 pathologic cases (of which 2 cases contained 2 pathology types and 1 case contained 3 pathology types), including 7 traumatic or complex injuries, 2 congenital malformations, 12 strokes/hemorrhages, 2 vascular malformations, 32 neoplasms/primary neoplastic cysts, 10 infectious/ demyelinating conditions, and 2 metabolic/degenerative disorders. Readers of synthetic MR imaging showed equal or higher ability to diagnose all pathologies, except for neoplasms/primary neoplastic cysts ( $n=2$, difference in detection of $\pm 6.3 \%$ sensitivity and $\pm 1.3 \%$ specificity among readers) subgroup and infectious diseases $(n=10$, difference in detection of $\pm 10.0 \%$ sensitivity and $\pm 3.0 \%$ specificity).

\section{DISCUSSION}

To our knowledge, this is the first large, prospective, randomized study of synthetic MR imaging technology to enroll a cross-section of the neuroimaging population, including a variety of brain pathologies encountered in clinical practice. On the basis of blinded assessments from 7 neuroradiologists, the overall diagnostic quality of synthetic MR imaging was statistically noninferior to conventional MR imaging series for T1- and T2-weighted, T1 and T2 FLAIR, STIR, and PD contrast views. Furthermore, neuroradiologists reported similar anatomic/morphologic feature legibility in both synthetic and conventional images. Both synthetic and conventional sequences exhibited similar quality issues and artifact trends for T1- and T2-weighted, STIR, and PD contrast views, while synthetic imaging had more FLAIR artifacts. Synthetic FLAIR artifacts were readily recognizable by cross-comparison within contrast views and thus did not significantly impact the diagnostic use of synthetic MR imaging. Overall, study results demonstrated that both synthetic and conventional imaging have similar diagnostic utility.

Anatomic and morphologic characteristics were visible in AJNR Am J Neuroradiol 38:1103-10 Jun 2017 www.ajnr.org 1107 


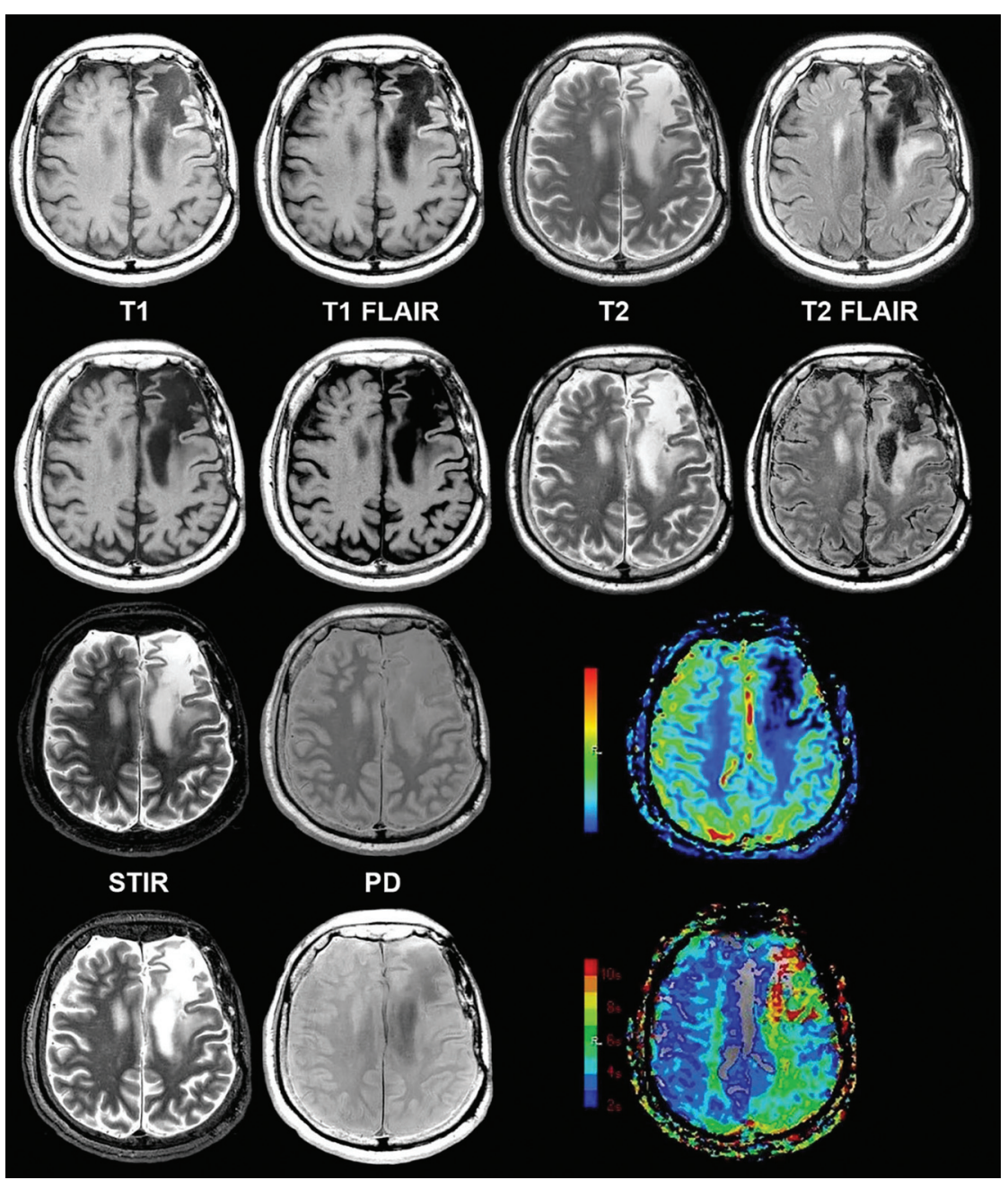

FIG 5. Chronic infarction in synthetic and conventional $3 T$ MR imaging shown alongside color functional perfusion maps in a 62-year-old man. Conventional (rows 7 and 3 ) and synthetic (rows 2 and 4) views show similar legibility and quality. T1 FLAIR and T2 FLAIR views have some granulated white noise in the margins. Color quantitative perfusion maps (lower right) demonstrate decreased flow and prolonged transit time in this region of chronic infarction.

both synthetic and conventional views, though both exhibited issues pertaining to visualizing the craniocervical junction, CSF suppression, and pulsation artifacts that are well-documented in MR imaging. ${ }^{5,15-18}$ Synthetic imaging exhibited characteristic hyperintense artifacts in FLAIR views, corroborating previous reports that further work will be necessary before synthetically generated FLAIR views can fully replace conventional FLAIR in practice. ${ }^{5,6}$ While FLAIR artifacts contributed to lower overall image quality scores (because all views were considered in this composite primary end point), the overall impact of FLAIR artifacts on diagnosis was inherently limited by the nature of the synthetic views, in which immediate cross-comparison with other contrast views is possible. On rare occasions, encoding artifacts in FLAIR views could necessitate clinical workflow changes such as the addition of a single conventional scan; however, the impact on the patient's overall scan experience is offset by the time savings of the synthetic acquisition. Furthermore, motion and signalencoding artifacts were observed to affect all reconstructed synthetic views if present in the original acquisition. As few as $7.5 \%$ of single MR images exhibited motion artifacts, while up to $19.8 \%$ of long scans of multiple contrasts may be affected. ${ }^{19}$ Because synthetic imaging reduces the overall scan time, the impact of acquisition issues is expected to be limited in practice.

Diagnostic performance of synthetic imaging was similar to that of conventional MR imaging, as indicated by statistical noninferiority of synthetic images. While the noninferiority model is decisive for effectiveness in therapeutic studies, which directly assess ultimate patient outcomes, elucidating the clinical implications of noninferiority findings in radiology is less straightforward because the negative effects of image quality may have variable effects on ultimate patient outcomes. ${ }^{14}$ Thus, from a clinical perspective, we observed that in both synthetic and conventional MR imaging, some neoplasms/primary neoplastic cysts and infectious or demyelinating conditions were challenging for readers to identify without additional clinical or laboratory work-up, possibly due to overlapping appearances of neoplastic and inflammatory conditions on MR images. ${ }^{20,21}$

The sensitivity and specificity of MR imaging in neuroradiology have been reported to range from $39 \%$ to $98 \%$ and $33 \%$ to $100 \%$, respectively, with wide variations based on reader experience and the pathologic condition studied. ${ }^{22-25}$ Across study readers, synthetic MR imaging sensitivity and specificity had values within typical clinically observed ranges for blinded MR imaging reads (without clinical context). ${ }^{22-25}$ Statistical variations in diagnostic classifications may be centrally attributable to small samples of certain pathologies in the present study, meriting further study of these pathologic subgroups. Synthetic scanning is performed in the axial view only, and some clinical cases may be limited by spatial resolution in this section direction. Owing to the relatively shorter synthetic acquisition time, however, additional sequences can also be combined with the synthetic acquisition in a single examination session with minimal burden on the patient.

The strengths of this study include the use of a prospective acquisition protocol with matched scanning parameters (On-line Table 1). Because scans were acquired in a fixed order with MDME (synthetic reconstruction) acquired last, a relative propensity toward motion artifacts in synthetic images may not be representative of actual occurrence. Reports have, however, shown that single scans of short duration have lower incidences of motion than longer scans. ${ }^{13,26}$ The trial results support the use of synthetic MR imaging in brain imaging to reduce scan time and 


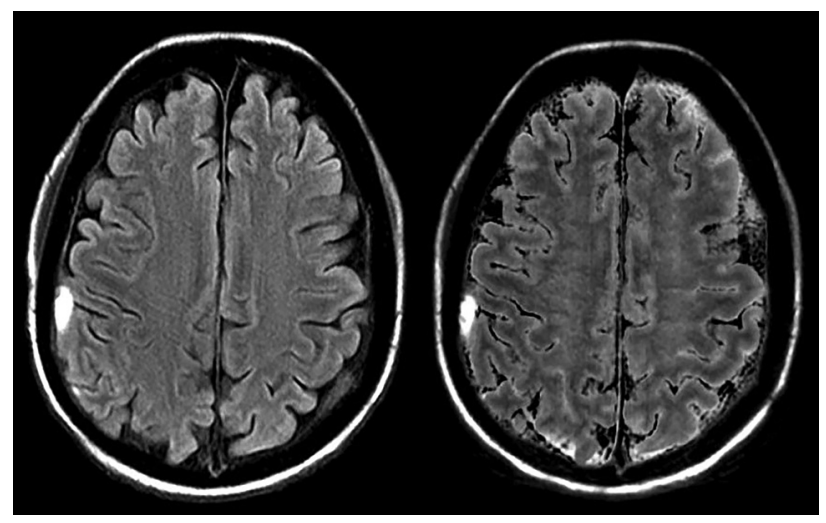

FIG 6. Subdural hematoma on T2 FLAIR in synthetic and conventional $3 T$ MR imaging demonstrating pronounced artifacts. Conventional (left) and synthetic (right) T2 FLAIR images are shown for a patient with subdural hematoma, in which synthetic T2 FLAIR has notable granulated hyperintensities and lacks contrast between the lesion and surrounding tissues. Artifacts of this severity level were rare among synthetically reconstructed images, possibly due to issues in the MDME acquisition that are typically resolved on rescanning. For cases demonstrating these granulated hyperintensities on the synthetic T2 FLAIR, artifacts were readily recognizable by characteristic distortion and correlation with other contrast views without apparent artifacts. While these could necessitate rescanning with conventional T2 FLAIR in some cases, when coupled with other contrast views, these artifacts did not interfere with the diagnostic accuracy of synthetic MR imaging.

the associated discomfort for patients undergoing brain MR imaging, with diagnostic performance similar to that of conventional imaging.

\section{CONCLUSIONS}

The current study demonstrated that synthetic images were statistically noninferior in terms of overall diagnostic image quality compared with conventional MR images, with similar diagnostic utility for detecting a range of brain pathologies. Both synthetic and conventional MR imaging could visualize anatomic and morphologic features of the brain, with similar trends in artifacts and diagnostic utility. Because synthetic reconstructions rely on the quality of a single scan, care should be taken to minimize motion and acquisition artifacts. While more artifacts were observed in synthetic T2 FLAIR reconstructions, cross-comparison with other contrast views enabled neuroradiologists to readily detect these artifacts without interfering with the diagnostic ability of synthetic images. The trial results support the use of synthetic MR imaging in brain imaging to reduce scan time and discomfort for patients undergoing brain MR imaging, while acquiring highquality diagnostic MR images. We expect that further research may reveal additional applications for synthetic MR imaging.

\section{ACKNOWLEDGMENTS}

We thank the participants of the study, the study coordinators across multiple sites, and the study readers. We also acknowledge the contributions of Dr Michael Starc (Department of Radiology, Weill Cornell Medical College, New York, NY) in study conduct and of Dr Keyi Wang (GE Healthcare, Technology and Medical Innovation) for assistance with the statistical design of the trial.

Disclosures: Lawrence N. Tanenbaum—RELATED: Grant: GE Healthcare, Comments: sponsored trial payments for patient scanning*; UNRELATED: Consulting Fee or Honorarium: GE Healthcare; Consultancy: GE Healthcare. Angela N. Johnson-RELATED:
Support for Travel to Meetings for the Study or Other Purposes: GE Healthcare, Comments: Funding for travel was provided by GE Healthcare for US FDA agency and investigator meetings associated with study conduct. No additional funding was provided for the publication activities; UNRELATED: Employment: GE Healthcare, Comments: Angela N. Johnson is an employee of the Technology and Medical Innovation Organization of GE Healthcare. Providing publication assistance is not a requirement of employment or incentivized by GE Healthcare; Payment for Lectures Including Service on Speakers Bureaus: Regulatory Affairs Professional Society, Comments: conference faculty for the annual conference, receiving honorarium; Stock/Stock Options: GE Healthcare, Comments: I hold employee stock options in GE Healthcare; OTHER RELATIONSHIPS: I am a doctoral student at Texas Tech University receiving employee-sponsored tuition benefits for doctoral research. Ajit Shankaranarayanan—UNRELATED: Employment: GE Healthcare. Mathias GoyenUNRELATED: Employment: GE Healthcare, Comments: full-time employment, Chief Medical Officer Oncology; Stock/Stock Options: GE Healthcare. Aaron S. FieldRELATED: Grant: GE Healthcare, Comments: grant from study sponsor to cover institutional costs associated with participation in the trial. This did not include any salary or other support paid to me*. ${ }^{*}$ Money paid to the institution.

\section{REFERENCES}

1. Riederer S, Suddarth S, Bobman S. Automated MR image synthesis: feasibility studies. Radiology 1984;153:203-06 CrossRef Medline

2. Warntjes J, Dahlqvist O, Lundberg P. Novel method for rapid, simultaneous T1, T2*, and proton density quantification. Magn Reson Med 2007;57:528-37 CrossRef Medline

3. Krauss W, Gunnarsson M, Andersson T, et al. Accuracy and reproducibility of a quantitative magnetic resonance imaging method for concurrent measurements of tissue relaxation times and proton density. Magn Reson Imaging 2015;33:584-91 CrossRef Medline

4. Warntjes J, Leinhard O, West J, et al. Rapid magnetic resonance quantification on the brain: optimization for clinical usage. Magn Reson Med 2008;60:320-29 CrossRef Medline

5. Blystad I, Warntjes J, Smedby O, et al. Synthetic MRI of the brain in a clinical setting. Acta Radiol 2012;53:1158-63 CrossRef Medline

6. Granberg T, Uppman M, Hashim, et al. Clinical feasibility of synthetic MRI in multiple sclerosis: a diagnostic and volumetric validation study. AJNR Am J Neuroradiol 2016;37:1023-29 CrossRef Medline

7. Hagiwara A, Hori M, Suzuki M, et al. Contrast-enhanced synthetic MRI for the detection of brain metastases. Acta Radiol Open 2016;5: 2058460115626757 CrossRef Medline

8. Betts M, Leach J, Jones B, et al. Brain imaging with synthetic MR in children: clinical quality assessment. Neuroradiology 2016;58: 1017-26 CrossRef Medline

9. Struck A, Westover M. Variability in clinical assessment of neuroimaging in temporal lobe epilepsy. Seizure 2015;30:132-35 CrossRef Medline

10. Wattjes M, Wijburg M, Vennegoor A, et al. Diagnostic performance of brain MRI in pharmacovigilance of natalizumab-treated MS patients. Mult Scler 2016;22:1174-83 CrossRef Medline

11. Gulani V, Schmitt P, Griswold M. Towards a single-sequence neurologic magnetic resonance imaging examination: multiple-contrast images from an IR TrueFISP experiment. Invest Radiol 2004; 39:767-74 CrossRef Medline

12. Osborn AG, Salzman KL, Barkovich AJ. Diagnostic Imaging: Brain. 2nd ed. Friesens: Amirsys; 2010

13. Krupa K, Bekiesińska-Figatowska M. Artifacts in magnetic resonance imaging. Pol J Radiol 2015;80:93-106 CrossRef Medline

14. Ahn S, Park SH, Lee KH. How to demonstrate similarity by using noninferiority and equivalence statistical testing in radiology research. Radiology 2013;267:328-38 CrossRef Medline

15. Tha K, Terae S, Kudo K, et al. Differential diagnosis of hyperintense cerebrospinal fluid on fluid-attenuated inversion recovery images of the brain, Part II: non-pathological conditions. Br J Radiol 2009; 82:610-14 CrossRef Medline

16. Tha K, Terae S, Kudo K, et al. Differential diagnosis of hyperintense cerebrospinal fluid on fluid-attenuated inversion recovery images of the brain, Part I: pathological conditions. Br J Radiol 2009;82: 426-34 Medline 
17. Ogbole G, Soneye M, Okorie C, et al. Intraventricular cerebrospinal fluid pulsation artifacts on low-field magnetic resonance imaging: potential pitfall in diagnosis? Niger Med J 2016;57:59-63 CrossRef Medline

18. Hakky M, Pandey S, Kwak E, et al. Application of basic physics principles to clinical neuroradiology: differentiating artifacts from true pathology on MRI. Am J Roentgenol 2013;201:369-77 CrossRef Medline

19. Andre J, Bresnahan B, Mossa-Basha M, et al. Toward quantifying the prevalence, severity, and cost associated with patient motion during clinical MR examinations. J Am Coll Radiol 2015;12:689-95 CrossRef Medline

20. Dagher AP, Smirniotopoulos J. Tumefactive demyelinating lesions. Neuroradiology 1996;38:560-65 CrossRef Medline

21. Mabray M, Cohen B, Villanueva-Meyer J, et al. Performance of apparent diffusion coefficient values and conventional MRI features in differentiating tumefactive demyelinating lesions from primary brain neoplasms. AJR Am J Roentgenol 2015;205:1075-85 CrossRef Medline
22. Atighechi S, Zolfaghari A, Baradaranfar M, et al. Estimation of sensitivity and specificity of brain magnetic resonance imaging and single photon emission computed tomography in the diagnosis of olfactory dysfunction after head traumas. Am J Rhinol Allergy 2013; 27:403-06 CrossRef Medline

23. Wollman D. Sensitivity and specificity of neuroimaging for the diagnosis of Alzheimer's disease. Dialogues Clin Neurosci 2003;5: 89-99 Medline

24. Sabol Z, Resić B, Juraski R, et al. Clinical sensitivity and specificity of multiple $\mathrm{T} 2$-hyperintensities on brain magnetic resonance imaging in diagnosis of neurofibromatosis type 1 in children: diagnostic accuracy study. Croat Med J 2011;52:488-96 CrossRef Medline

25. Polman C, Reingold S, Banwell B, et al. Diagnostic criteria for multiple sclerosis: 2010 revisions to the McDonald criteria. Ann Neurol 2011;69:292-302 CrossRef Medline

26. Zaitsev M, Maclaren J, Herbst M. Motion artifacts in MRI: a complex problem with many partial solutions. J Magn Reson Imaging 2015; 42:887-901 CrossRef Medline 